\title{
A new mechanism controlling conduction in stretched myelinated nerves and a comprehensive nerve conduction model
}

\author{
K Siddique-e Rabbani \\ Department of Biomedical Physics \& Technology, University of Dhaka, Dhaka, Bangladesh \\ Email: rabbani@du.ac.bd
}

\begin{abstract}
Analysing published experimental findings this paper revealed that for myelinated nerves the conduction velocity $(C V)$ increases on stretching out of the nerve, which has not been pointed out by anyone before. This apparently contradicts existing concepts since stretching out of a nerve fibre reduces its diameter which is expected to reduce the $C V$. Besides, the change is reversible and immediate, which cannot be explained with existing knowledge either. In order to explain this anomaly, the present work invoked a new resistance to ion flow between the nerve axon and the extracellular fluid created by interdigitated fingerlike processes of myelin sheaths coming from two sides of a node of Ranvier, analyzing published electron microscopic images. When stretched out, the gaps between the processes increase, decreasing the resistance to ion flow and thereby hastening depolarization, increasing $C V$ in turn. The gaps close immediately on the release of the stretching force because of the pull of the elastic endoneurium, thus retrieving the original $C V$. To represent this new mechanism, a new resistive element has been added to the existing electrical model of a myelinated nerve, which is being claimed to be the dominant component that determines the conduction delay. Stretching also affects other nerve parameters and this paper developed a mathematical formulation involving all these parameters to show satisfactorily that $C V$ indeed increases with stretching, in which the contribution of the proposed resistance dominates. The paper also proposed an appropriate modification of the representative schematic model commonly used to depict propagation of action potential in a myelinated nerve fibre. The suggested new mechanism and the resistance model is a breakthrough in the explanation of neural conduction and opens up the door for new study as well as for reviewing all previous experiments on myelinated nerves afresh.
\end{abstract}

Keywords: Nerve conduction, neurophysiology, myelinated nerves, Node of Ranvier, RC model for nerve, comprehensive nerve conduction model. 


\section{INTRODUCTION}

Recent experiments, conceived and designed by the present author and carried out by his colleagues and students, indicate that conduction of action potentials through the Brachial Plexus are affected by simple head bending, and that the changes are immediate [Rahman 2008, Sharmin and Rabbani 2016]. The changes again revert back to original value immediately when the head is brought back to normal upright position. These immediate changes could not be explained based on existing knowledge of nerve conduction mechanisms.

Detection of these changes, however, needed a new nerve conduction technique which was introduced by the author and his group as 'Distribution of F-latency (DFL)' [Rabbani et al 2007], obtained by multiple electrical stimulation (30 to 40 times) of a peripheral nerve and recording the latencies of a secondary evoked EMG, called the F-wave, from a supplied muscle. The F-latencies (time taken from stimulation to onset of the F-response) vary randomly on multiple stimulations, given adequate intervals such that the physical conditions stabilize before each stimulation. One major contribution of this research group was in establishing a physiological relationship between 'Distribution of Conduction velocity (DCV)' of large motor fibres that contribute to F-responses and DFL, through logical arguments. This group established that DCV of such large motor fibres determines DFL and that the relative DCV can be obtained approximately as the mirror image of DFL where the latter can be measured very simply in a clinic using conventional EMG techniques. DCV is a well sought-after parameter but no clinically suitable techniques to obtain DCV existed before this method based on DFL.

Since F-responses are known to occur due to random recruitment of different nerve fibres, which again have contributions from different nerve roots, an individual F-latency from the median nerve cannot give any information on conduction changes at the brachial plexus or at the nerve roots. On the other hand, DFL, being a statistical phenomenon of the collection of a multiple of F-responses, appeared to be sensitive to such changes [Alam and Rabbani 2010, Rabbani 2011]. In these works, DFL obtained from the median nerve was found to be sensitive to cervical spondylotic neuropathy (radiculopathy or myelopathy at the relevant roots or vertebral segments respectively) [Rahman et al 2013, Rabbani et al 2014]. Later improvements were found to give about $80 \%$ correct prediction of such pathologies with respect to MRI findings [Chowdhury et al 2014]. Similar results were also found in the case of lumbo-sacral pathologies by the author's group but a detailed clinical study is yet to be performed. 
Coming back to head bending experiments, significant delay shifts in the patterns of DFL with head bending indicated with confidence that there were changes in the conduction velocity of the nerve at the Brachial Plexus due to such manoeuvres, but the reasons could not be readily identified. Besides, the changes in DFL depend on the pathological condition of the nerves in the cervical region, which may give different results for different persons. However, these studies using DFL confirmed that changes in nerve conduction occurred due to head bending which were immediate, and these values returned to the original on bringing the head position back to normal again.

The above experimental findings led to further query and a literature survey revealed that immediate changes in nerve conduction were reported by at least two groups working to determine the effect of elbow bending on ulnar nerve conduction velocity (NCV), where the outcomes are direct and easier to interpret. Harding \& Halar [1983] and Sattari \& Emad [2007] independently measured the NCV in the ulnar nerve for a $10 \mathrm{~cm}$ segment across the elbow for different angles of bending of the hand, taking reasonable sizes of sample populations and came up with similar results. Sattari \& Emad [2007] measured both motor and sensory NCV along a $10 \mathrm{~cm}$ section across elbow bilaterally in 50 able-bodied subjects (100 nerves on two sides) for flexing angles of $0^{\circ}, 45^{\circ}, 90^{\circ}, 110^{\circ}$ and $135^{\circ}$ (full extension of the hand considered as $0^{\circ}$ ). The mean motor NCV was $54 \mathrm{~m} / \mathrm{s}$ at full extension of the hand $\left(0^{0}\right.$ flexion of the elbow $)$ while it increased to $63.4 \mathrm{~m} / \mathrm{s}$ for a flexion of $135^{\circ}$, an increase of about $17 \%$, the changes being systematic over the range. For the same manoeuvre the mean sensory NCV of the same segment changed from $56.7 \mathrm{~m} / \mathrm{s}$ to $67 \mathrm{~m} / \mathrm{s}$, an increase of about $18 \%$, almost the same as for the motor nerve.

Therefore, it was evident that the NCV increased systematically and significantly when the hand was flexed through different angles from a full extended position; which was immediate and reversible. However, none of the above works, carried out with a time interval of more than 30 years, could identify the reasons for the apparent changes observed, nor they attempted for such an explanation. These works were aimed at finding the best angle for clinical measurements to give the same NCV using above-elbow or below-elbow stimulation and suggested that possible inaccuracies in measurements of nerve lengths were responsible for the observed changes, although the measurements were carried out with great care on a sizeable sample size (40 limbs in the former and 100 limbs in the latter). 
In an attempt to explain the above mentioned increase in NCV with flexion, the present work goes into the basics as described below. From an anatomical standpoint, flexion of the ulnar nerve at the elbow corresponds to stretching out of the nerve trunk. Since these experiments involve myelinated nerves only, these results suggest that for myelinated nerve fibres, $N C V$ increases with stretching out. This is an important observation which needs to be interpreted and analysed carefully, and from the viewpoint of the present author, it goes against prevalent knowledge and concepts of the mechanisms of nerve conduction, and is therefore an anomaly which needs to be resolved. This is further elaborated in the following paragraphs.

From a mechanical standpoint, stretching out of a soft tubular material such as the nerve will cause a reduction of the tube diameter. This has also been borne out for ulnar nerves by ultrasound scanning experiments by Thoirs et al [2008]. They measured the diameters of the ulnar nerve trunks at the level of the trochlear-coronoid articulation (which excluded the echogenic borders of the nerve) for two groups, normal subjects $(n=107)$ and patients with ulnar nerve entrapment (UNE) at the Cubital tunnel $(n=21)$. The mean nerve diameters for both groups decreased significantly from full extension to full flexion. For the normal population the mean diameter changed from $2.3 \mathrm{~mm}$ to $1.9 \mathrm{~mm}$ (about $20 \%$ ) for this manoeuvre, while that for patients with UNE changed from $2.5 \mathrm{~mm}$ to $2.2 \mathrm{~mm}$ (about 15\%). The overall larger diameters in UNE may be expected due to the pathology, but the direction of change on flexion is consistent with that of the normal fibres. For ulnar nerve passing through the Cubital tunnel at the elbow, as mentioned above, the nerve trunk is relaxed on extension and stretched out on flexion. Therefore, the findings clearly indicate that the diameter reduced on stretching out of a nerve fibre.

It needs to be pointed out that all the nerve conduction experiments mentioned in this study involved stretching of large diameter myelinated nerve fibres that are electrically stimulated through skin surface stimulation [Kimura, 1989, p.62-64]. Therefore, all the observations mentioned above, when combined, gives the suggestion that on stretching out of a myelinated nerve fibre, its diameter decreases and the corresponding conduction velocity increases.

The above mentioned observation on conduction velocity is apparently contrary to long established facts that conduction velocity of a nerve fibre increases with the diameter of the axon [Hursh 1939, Gasser and Grundfest 1939]. 
Therefore, all the above findings reveal the fact that there still exists a big anomaly in the explanation of the mechanism of nerve conduction, particularly for stretched myelinated nerves.

The above anomaly prompted the author to look into myelinated nerves in more detail through which he came up with some significant observations and explanations based on which he could resolve this anomaly with reasonable satisfaction. In fact, the proposals put forward in the present paper, based on fresh analyses of previously published experimental results, appear to make a breakthrough in the field of neurological science.

\section{Weakness of the traditional model for conduction in a myelinated nerve}

The model of a myelinated nerve fibre that is used for a traditional visualisation of its conduction is given in Figure 1 while an equivalent electrical model is given in Figure 2. Of course there are electrical models with varied complexity [Hodgkin and Huxley 1952, McIntyre et al 2002, Ladenbauer et al 2010, Kolaric et al 2013, Brown and Hamann 2014], but Figure 2 would be a typical model, particularly to describe the delay mechanism for the propagation of action potentials. It needs to be pointed out that for the explanation of conduction in nerve fibres, the main focus should be on the phase in which the transmembrane potentials are still below the threshold that triggers a rapid depolarization through opening of the ion channels at the nodes. This means that the ion channels in the membranes are closed during this phase, which determines the conduction delay.

In the traditional visualization of a myelinated nerve fibre shown in Figure 1, points A, B and $\mathrm{C}$ indicate successive nodes. Figure 2 shows the electrical model between the intracellular and

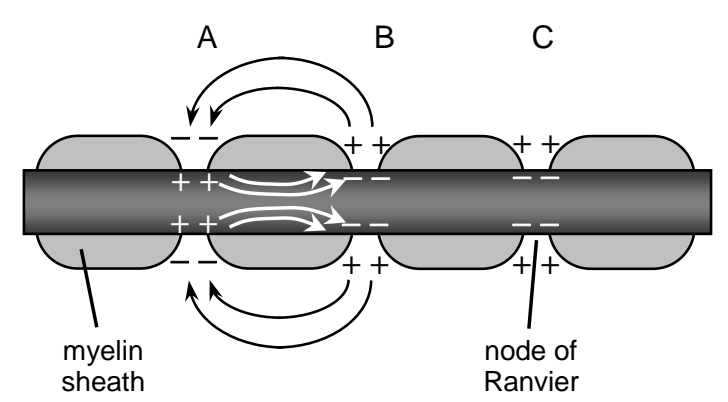

Fig. 1: Traditional visualization of a myelinated nerve fibre. Node of Ranvier has clear opening to extracellular fluids

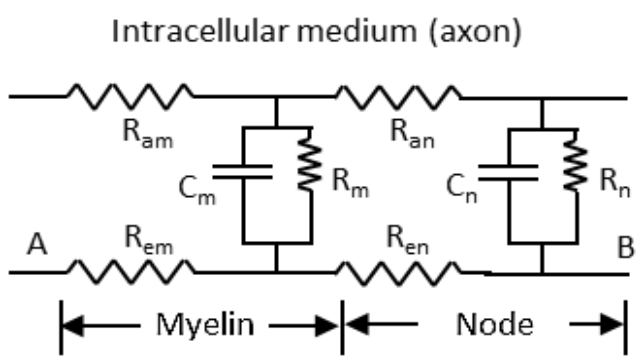

Extracellular medium

Fig. 2: Traditional electrical model of a myelinated nerve fibre 
extracellular mediums where the nodes $\mathrm{A}$ and B are also indicated at approximate representative points. Here $R_{a m}$ represents the axonal resistance between the nodes A \& B while $R_{m}$ and $C_{m}$ represent the effective leakage resistance and capacitance

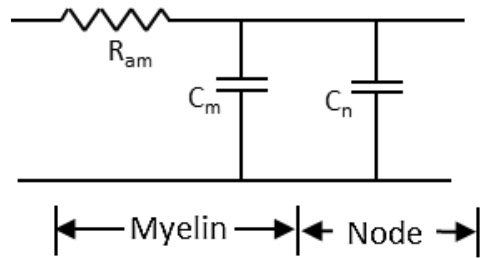

Fig. 3: Simplified traditional electrical model of a myelinated nerve fibre respectively of the myelin sheath and the axonal membrane of this internodal region. Typically $R_{m}$ would be very high as the fatty myelin sheath is essentially an insulator, and the leakage resistance of the membrane would be very high as well, since ion channels within this region are very sparse and remain inactive always. The extracellular resistance (outside the nerve axon) between the nodes A and B is represented by $R_{e m}$, which is expected to be low because of the presence of the fluid medium surrounding the nerve fibre. The nodal region is similarly represented by an axonal resistance $R_{a n}$ and an extracellular resistance $R_{e n}$. Since the length of the nodal region is very small (1 to $2 \mu \mathrm{m}$ ) and about a thousand times less than that of a myelinated segment (1 to $2 \mathrm{~mm}$ ) [Keynes et al, 2011], $R_{a n} \ll<R_{a m}$. Similarly, $R_{e n} \ll<R_{e m} . R_{n}$ and $C_{n}$ represent the leakage resistance and capacitance respectively of the axonal membrane in the nodal region. The axonal membrane is basically an insulating lipid layer except for the ion channels when these are open. Therefore, $R_{n}$ should be very high when the ion channels in the axonal membrane are closed, which is the phase under discussion here. In view of the relative magnitudes of the parameters, a simplified circuit model may be drawn for delay analysis, as shown in Figure 3. This shows that the time constant of the delay for propagation of nerve action potentials per node would be essentially given by,

$$
\tau=R_{a m}\left(C_{m}+C_{n}\right)
$$

So when depolarization occurs at a particular node, the time delay to depolarize the next node would depend on the above time constant.

Figure 4 shows schematics (not to scale) to indicate the relevant parameters of the myelinated segment and the nodal segment. Here,

$l_{n}=$ length of the nodal region

$t_{m}=$ thickness of the myelin sheath

$t_{n}=$ thicknes of the axonal membrane

$D=$ diameter of the axon. 
Other relevant parameters are:

$l_{m}=$ inter-nodal nerve length (of the myelinated region, not shown)

$\varepsilon_{m}=$ permittivity of the myelin sheath

$\varepsilon_{n}=$ permittivity of the axonal

membrane

In terms of the above, the parameters in Equation 1 are:

$R_{a m}=\rho l_{m} /\left(\pi D^{2} / 4\right)$

$C_{m}=\varepsilon_{m}\left(\pi D l_{m}\right) / t_{m}$

$C_{n}=\varepsilon_{n}\left(\pi D l_{n}\right) / t_{n}$

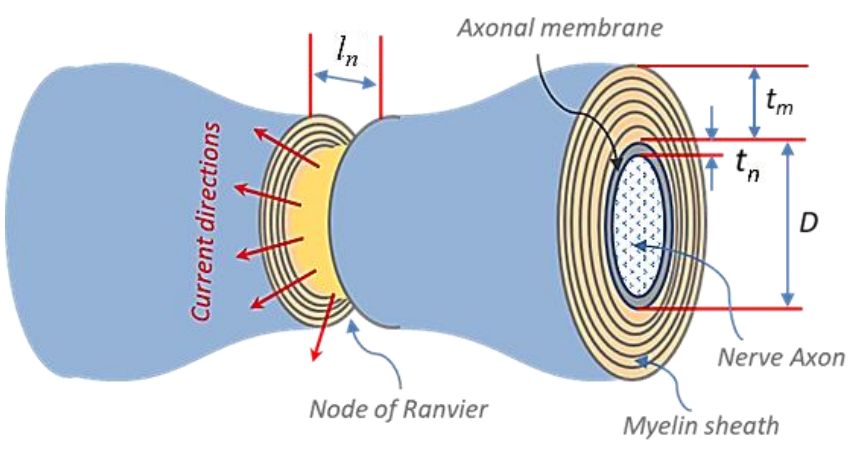

Fig. 4: Illustrating representative parameters for time constant estimation and radial current at the node that contributes to the time constant for depolarisation.

In the myelinated region, $C_{m}$ has contribution from both the myelin sheath and the axonal membrane, the capacitances appearing in series. Since $\mathrm{t}_{\mathrm{m}} \gg \mathrm{t}_{\mathrm{n}}$ and $\varepsilon_{m} \& \varepsilon_{n}$ are expected to be of the same order, capacitance due to the myelin sheath will be very small compared to that due to the axonal membrane. In series combination of two capacitances it is the smaller one that dominates (has larger reactance), therefore, the contribution of the axonal membrane has been ignored in Equation 3. Consideration of all the equations above will show that the time constant $\tau$ will be inversely proportional to diameter $D$ if other parameters remain constant. Therefore, axonal conduction velocity $C V$, which would be inversely proportional to the time delay, will be directly proportional to the diameter $D$, supporting the age old experimental results mentioned before.

However, as mentioned in the previous section, this model contradicts reported experimental findings highlighted in this work that stretching out of a myelinated nerve increases the $C V$, as stretching would cause a decrease in the diameter. This, therefore, poses an anomaly which has not been indicated nor explained by anyone so far.

Studying published images of the node of Ranvier taken using electronic microscopes, the present work attempts to explain this new observation that $C V$ increases with stretching of a myelinated nerve fibre. In doing so the present work introduced a new resistance parameter, which would play the dominant role in the determination of conduction velocity in a myelinated nerve fibre and would explain the anomaly mentioned above. This model also should preserve 
the established knowledge that for nerve fibres of the same type with different diameters, $C V$ is proportional to the axonal diameter. The present work also attempts a mathematical formulation to include relevant nerve parameters to explain why $C V$ of a myelinated nerve fibre increases on stretching out. Thus the present work attempts to come up with a new nerve conduction model, which would be a comprehensive one, having the merit to replace the existing one.

\section{METHODS}

\section{The proposed model based on anatomical concepts}

The above anomaly led to a detailed study of the anatomy of the myelinated axons by the present author, particularly focusing at images of the node of Ranvier obtained using electron microscope [Porter and Bonneville 1973] and simplified visualization models [Camizu, downloaded 2018, Classroom.sdmesa.edu, downloaded, 2018]. Based on the above information, schematic diagrams of the axon and the junction of myelin sheaths around the

\section{Relaxed Nerve}

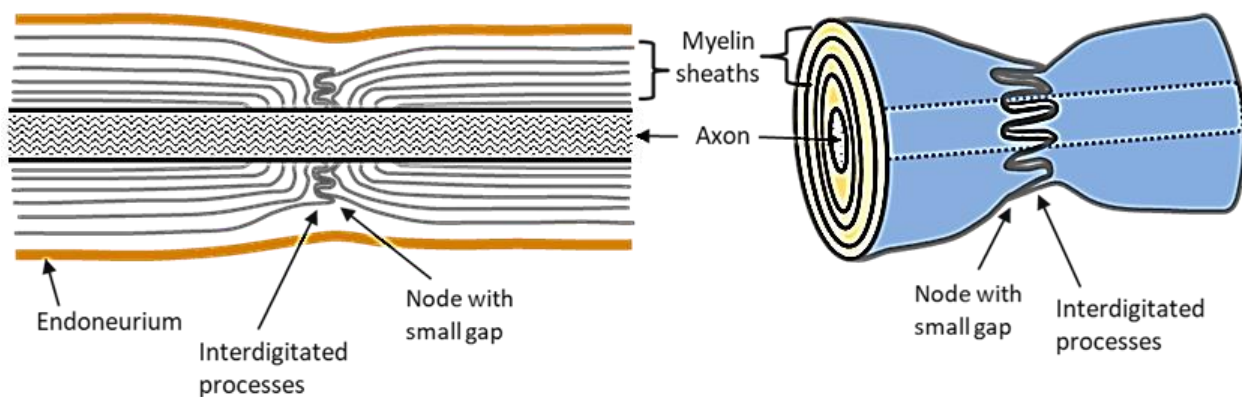

Stretched Nerve
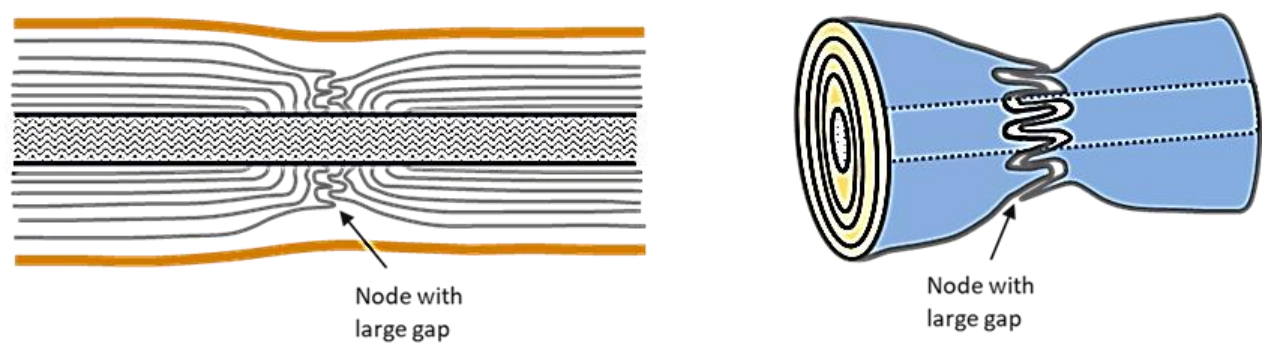

Fig.5: Schematic diagrams of a relaxed and a stretched out myelinated nerve fibre showing the varying gaps at the node of Ranvier, created by the interdigitated fingerlike processes of the Myelin sheaths on the two sides of the node. 
node of Ranvier are redrawn in Figure 5, for both unstretched (or relaxed, top diagrams) and stretched (bottom diagrams) conditions; visualization of the difference being the author's own. The left hand diagrams in this figure show $2 \mathrm{D}$ cross sections along the centre of a nerve fibre under the two conditions while the right hand diagrams show the corresponding 3D perspectives, but without the endoneurium. As can be seen, the myelin sheaths make a series of liplike folds in the extended margins near the node, covering the nerve axon. In the region of the node itself, fingerlike process of these neighbouring myelin sheaths interdigitate and cover the nodal area in both longitudinal and transverse directions. The whole assembly is then wrapped around by the basement membrane and the endoneurium. This endoneurium is composed of connective tissue fibres having elastic properties and it protects the axons from possible mechanical damage. It also isolates electrical signals between adjacent nerve fibres. Therefore, it may easily be envisaged that the elastic pull of the endoneurium will tend to keep the myelinated segments on two sides of a node as close as possible, making a sort of snap-fit through the interdigitated fingerlike processes. The present author argues that in doing so, it will obstruct the flow of ions at the nodal region between the immediate outside of the axonal membrane and the extracellular fluid, which is required for depolarizing the nerve membrane; the ions will have to wind their way through the narrow zigzag paths around the fingerlike processes. Therefore, the time delay involved in depolarizing a node will heavily depend on this resistance to ion flow. Now, if the nerve is stretched out by external forces, the interdigitated fingerlike processes of the myelin sheaths on the two sides of the node will move away from each other increasing the gap between them (Fig. 5, bottom figures), which will decrease the above resistance to the flow of ions. Therefore, the time delay for depolarization of the node will be reduced contributing to an increase in the $C V$ of the nerve segment. On releasing the stretching force, the gap will close because of the elastic pull of the connective tissues of the endoneurium resulting in an immediate increase of the time delay for depolarization of the node decreasing the $C V$ back to the original value. Thus this apparently explains the immediate changes in $C V$ as observed experimentally.

Based on the above visualisation, the author puts forward the suggestion that the resistance to movement of ions around these interdigitated fingerlike processes would contribute to a major part of the time delay of propagation of nerve action potentials, which no one has thought of before, and which would explain the increase in CV on stretching out, as observed in published experiments referred to in this paper. This also explains why the changes are immediate. 
Based on this suggestion the author proposes a modified electrical model of a myelinated nerve fibre as shown in Figure 6, where he has introduced a new resistance $R_{n e}$ between the outside of the nodal membrane and the extracellular fluid. The new proposed parameter $R_{n e}$ represents the resistance offered by the interdigitated fingerlike processes at the node to ion flow between the nodal membrane and the extracellular fluid. However, it is the potential across $C_{n}$ that is of importance here, which has to reach the threshold for depolarization; for subsequent propagation of action potentials. Figure 7 shows a simplified model following the assumptions put forward earlier for Figure 3.

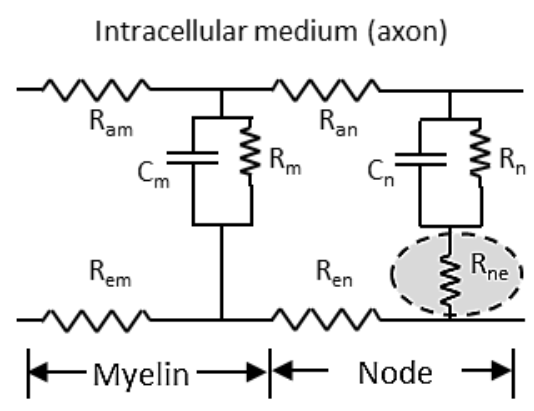

Extracellular medium

Fig. 6: Proposed electrical conduction model of a myelinated nerve fibre. Here $R_{n e}$ is the proposed addition.

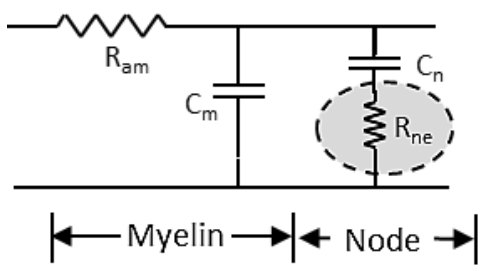

Fig. 7: Simplified version of the proposed electrical conduction model of a myelinated nerve fibre.

\section{Explaining increase in CV due to stretching out of myelinated nerve fibres}

In Equations 1 to 4 and in Figure 7, some ideas on the magnitudes of values some of the relevant parameters would be useful in visualising the relative effects of the individual components. Assuming typical values as (illustrated in Figure 4, except $l_{m}$ ): $l_{m}=1000 \mu m, l_{n}=1 \mu m$, $D=10 \mu m, t_{m}=2.5 \mu m, t_{n}<10 \mathrm{~nm}$, and assuming $\varepsilon_{m}$ and $\varepsilon_{n}$ to be the same, $C_{m}$ and $C_{n}$ will be of similar order which has also been indicated by Ritchie (1995). The author also suggests that $R_{n e}>>R_{a m}$ because the axon has a diameter of around $10 \mu \mathrm{m}$ while the gaps of the interdigitated fingerlike processes at the node will be much smaller, expected to be in the range of tens of $\mathrm{nm}$. Besides, the ions have to move across many bends and obstacles increasing the effective length.

Here the potential across $C_{n}$ is the transmembrane potential that plays the main role in depolarization of the nodal region, contributing to the $C V$ of the nerve fibre. Since $C_{m}$ and $C_{n}$ 
are of similar order and $R_{n e}>>R_{a m}$, the time delay would be dominated by the new time constant (reference: Figure 7)

$$
\tau_{n}=R_{n e} C_{n}
$$

Therefore, it is the change in this time constant that would contribute to the change in $C V$ on stretching of a myelinated nerve fibre.

When the nerve fibre is stretched, the gaps between the interdigitated fingerlike processes will increase, reducing the value of $R_{n e}$. Therefore the time constant $\tau_{n}$ will tend to decrease resulting in an increased $C V$. However, there may also be a change in $C_{n}$ due to the stretching, and a combined analysis needs to be made in order to obtain a proper inference.

Figure 8 shows a simplified
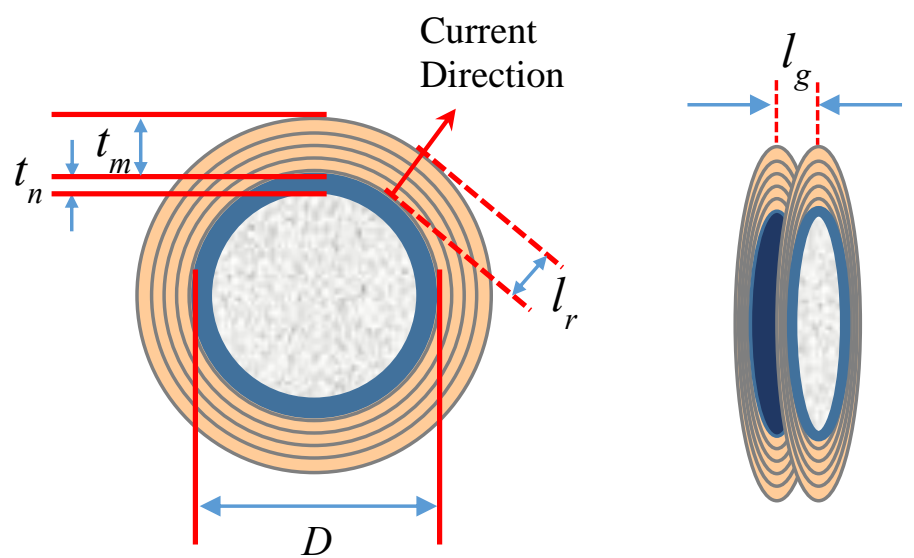
diagram of Figure 8 . The central shaded zone represents the axon with a diameter $D$ and its membrane Fig. 8: Geometry for estimating resistance $R_{n e}$ and $C_{n}$ at the node of Ranvier, at the gap between myelin lips with a thickness $t_{n}$. The ringed zone outside represents the interdigitated lips of the myelin sheath with an overall thickness $t_{m}$. The right hand diagram shows an exaggerated perspective of the gap between the two sets of interdigitated lips of the myelin sheath from two sides, meeting at the node of Ranvier leaving gap width $l_{g}$. Of course in this simplified diagram the complex protrusions of the interdigitated lips are not shown. Here the gap width may be taken to equal the nodal length $l_{n}$ mentioned earlier. The gap would have a complex zig-zag pattern and is not expected to be the same all over the radial path; $l_{g}$ is simply representative of the fact that there is a gap through which ionic currents will flow. The gap width will be assumed to equal $l_{n}$ in subsequent analyses here. The ionic current responsible for depolarisation will be directed radially away from the axonal membrane as shown by the red arrow on the left hand diagram. The effective path length for the current is shown as $l_{r}$ which is shown in Figure 8 just to give an idea. However, the actual 
path length will be much longer because of the zig-zag path. Again, this length will decrease sharply with stretching as shown schematically in Figure 9. This decrease is expected to be much faster than a linear relationship in the beginning, saturating to a low value at larger stretching forces, but is not known at present.

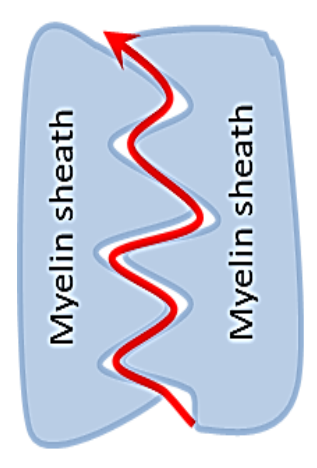

Unstretched

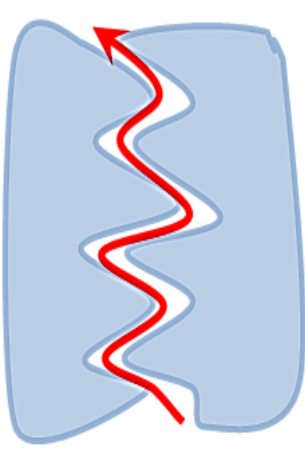

Mildly stretched

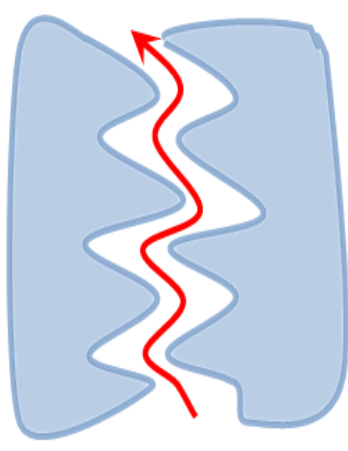

More stretched

Fig. 9: Radial path length $l_{r}$ for ionic current at node of Ranvier, between the outside of the membrane and the extracellular fluid through the narrow gap between interdigitated myelin sheaths, which is a zig-zag path, indicated by the red arrows. On increasing the gap due to stretching, the effective path length decreases considerably.

The right hand side of Equation 5 may be broken down to look for an overall dependence of the time constant on stretching of a nerve fibre. This is attempted below discussing the dependences of essential parameters.

The area offered to the current path at the nodal region increases with radius between the axonal membrane and outer edge of the myelin sheaths, therefore, the resistance offered by each radial layer will keep on decreasing. Assuming the gap $l_{n}$ to be constant over this region, the area would be proportional to the respective radius at each point and the resistance for an infinitesimally thin layer at any point would be inversely proportional to the radius. Although not accurate, but for simplicity, an effective average value of the area $A_{a v}$ corresponding to an effective average diameter $D_{a v}$ is considered for estimating the whole resistance $R_{n e}$. Thus, resistance $R_{n e}$ to radial current through an effective path length $l_{r}$ would be,

$R_{n e}=\rho l_{r} / A_{a v}$, where $\rho$ is the resistivity of the fluid medium in the gap region.

Now for the other component, the axonal membrane capacitance $C_{n}$ for the nodal region with membrane thickness $t_{n}$ is given by, 
$C_{n}=\varepsilon A / t_{n}$, where $\varepsilon$ is the permittivity of the membrane and $A$ is the area of the membrane at the nodal region.

Therefore, the time constant in equation 5 becomes,

$$
\tau_{n}=R_{n e} C_{n}=\rho \varepsilon A l_{r} / A_{a v} t_{n}
$$

Since $A / A_{a v}=D / D_{a v}$ the above equation may be rewritten as,

$$
\tau_{n}=R_{n e} C_{n}=\rho \varepsilon\left(D / D_{a v}\right)\left(l_{r} / t_{n}\right)
$$

As mentioned before, a nerve fibre is like a tube made of a soft elastic material. Therefore, on stretching of a myelinated nerve fibre, both $D$ and $D_{a v}$ are expected to decrease, and these will decrease by the same percentage. Therefore, $D / D_{a v}$, i.e., $A / A_{a v}$ may be assumed to remain unaltered on stretching of a myelinated nerve. Since $\rho$ and $\varepsilon$ are constants, the variation of $\tau_{n}$ on stretching may be expressed as,

$$
\tau_{n} \propto l_{r} / t_{n}
$$

Since conduction velocity $C V \propto 1 / \tau_{n}$, it may be written as,

$$
C V \propto t_{n} / l_{r}
$$

Which only depends on axonal membrane thickness and the radial path length for the ionic movement at the node. This equation provides the relationship for estimating the dependence of $C V$ on stretching of a myelinated nerve fibre.

On stretching, or, elongation of a nerve fibre, the membrane thickness $t_{n}$ is expected to decrease, and the percentage decrease is expected to be linearly related to the percentage increase in length of the nerve fibre for small extensions. On the other hand, the percentage change of the path length $l_{r}$ for the ions between the outside of the axonal membrane and the extracellular fluid outside the myelin sheath is expected to decrease at a

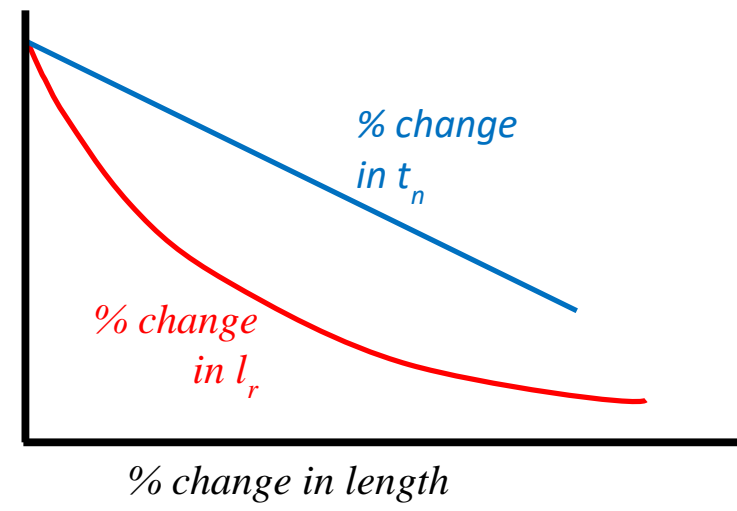

Fig. 10: Schematic decrease of $l_{r}$ and $t_{n}$ to visualize the behavior of change with stretching (elongation) of a fixed segment of a myelinated nerve fibre. Decrease of $l_{r}$ is much sharper than that of $t_{n}$. 
much faster rate, as suggested by Figure 9, and will saturate to a value equal to the thickness of the myelin sheath at the nodes at very large extensions. A schematic of the two variations are presented in Figure 10. At this point, it is not known how $l_{r}$ will change, but this diagram is presented just to aid visualization of the overall effect for the change in $C V$. It is clear from Figure 10 that although both $t_{n}$ and $l_{r}$ decrease with elongation of a nerve fibre, since $l_{r}$ decreases at a much greater rate, this will dominate the overall effect. Therefore, $C V$ will increase with stretching or elongation of a myelinated nerve fibre from its original relaxed condition. It may be possible that at a large extension the increase will be halted, but it is suggested that for practical stretching amounts, as observed in the experimental observations described in this paper, the phenomenon of increasing $C V$ will hold.

\section{Proposal of a schematic model of myelinated nerve fibres}

Based on the above observations and findings the present work proposes a simple visualization of nerve signal propagation in myelinated nerve fibres to replace the commonly used schematic model as given in Figure 1. The

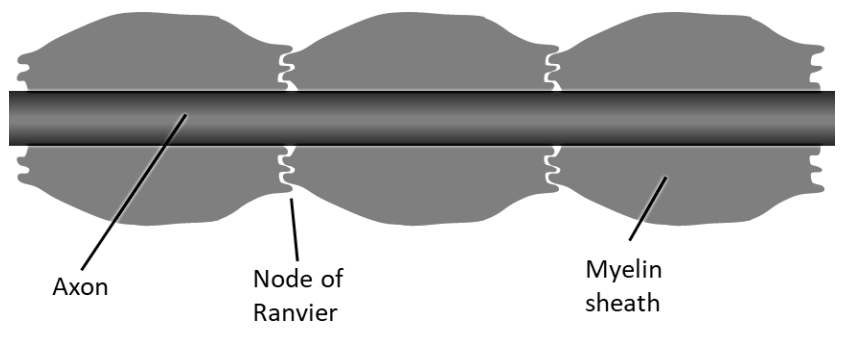

Fig.11: Suggested simple visualization model of a myelinated nerve conventional model gives a feeling that the node is virtually open to the extracellular fluid medium which is not the case as the present work suggests. Therefore, this visualization needs to be corrected and the author suggests a schematic visualization as given in Figure 11 which should be used instead. This model shows the resistance that ions face in moving between the nodal membranes and the extracellular fluids through a zig-zag path.

\section{RESULTS AND DISCUSSIONS}

Starting from a head bending experiment to observe quick changes in Distribution of F-Latency (DFL) [Rahman 2008, Sharmin and Rabbani 2016], where DFL is a new parameter introduced by the author's group [Rabbani et al 2007], the present work revealed an important scientific information analysing published experimental work on ulnar nerve [Harding \& Halar 1983, Sattari \& Emad 2007, Thoirs et al 2008] that conduction velocity (CV) of myelinated nerve increase on stretching and reverts back to original when the stretching force is withdrawn. Besides, these changes are immediate. None of these concepts exists in the present knowledge 
of neuroscience, so the present work had to obtain resources from several published works, logical arguments and mathematical models to establish the new viewpoint on a sound footing.

Of course the original authors of the above experimental work on ulnar nerve did not realise that their experiments caused stretching of the nerve. What the first two authors did was to measure the $C V$ of the ulnar nerve around the elbow on flexion of the hand through different angles to find an optimum angle for reproducible measurement, since it was well known that the measured $C V$ of the ulnar nerve across the elbow changes with angles of flexion. They had thought that these changes were due to physical movement of the ulnar nerve inside leading to errors in their measurements, in spite of the fact that the changes were systematic with angles of flexion, carried out on many nerves (40 and 100 respectively, in the two works). They were simply interested in some clinical procedures through these experiments and did not realise that some important information lay hidden. The third author provided the information that the diameter of ulnar nerve at the elbow decreased with flexion. Combining the above two information with a logical argument of his own, the present author suggested that the ulnar nerve at elbow gets stretched on flexion.

Analyses of the above published works allowed the present work to get to an important discovery that the CV of a myelinated nerve fibre increases on stretching, not known before. In order to explain this new finding the author had several achievements, reported in this paper, which may be summarized as follows.

i. From published images of the nodes of Ranvier obtained using electron microscopes, the present work suggested that interdigitated lips of myelin sheaths coming from two sides at nodes of Ranvier pose a resistance to ionic current flow between the outside of a nerve axon and the extracellular fluid, causing a delay in depolarization of the node that contributes to propagation of nerve action potentials. Previously this visualization was totally absent in this area of neuroscience.

ii. The present work introduced the above concept as a new resistance $R_{n e}$ appropriately to the conventional electrical model of a nerve fibre.

iii. The analysis incorporating the proposed resistance $R_{n e}$ shows that for a stretched myelinated nerve fibre, the $C V$ will increase in spite of a reduction in the diameter of the nerve fibre, which explains the anomalous behavior well.

iv. The proposed explanation also suggests that the changes in $C V$ on stretching will be immediate, explaining the experimental observations. 
v. The present work suggested that the above resistance $R_{n e}$ contributes to a time constant in conjunction with the capacitance $C_{n}$ of the axonal membrane in the nodal region, and this time constant is the dominant contributor to the overall time delay involved in the propagation of nerve action potentials. Previously, the time delay for propagation was thought to come from the axonal resistance $R_{a m}$ and the membrane capacitance $\left(C_{m}+C_{n}\right)$ only. This work suggests and shows through logical arguments that this previous time constant $R_{a m}\left(C_{m}+C_{n}\right)$ is negligible compared to the new time constant $R_{n e} C_{n}$.

vi. The present work introduced a mathematical model to analyse the contribution of different geometrical and electrical parameters in the delay time constant, contributing to a delay in propagation of nerve action potential. The $C V$ is proportional to the inverse of this time constant and the model successfully shows that $C V$ increases with stretching of a myelinated nerve fibre.

vii. The model also supports the conventional knowledge that $C V$ increases with diameter for a particular type of nerve fibre.

viii. The author suggests that this new model is a comprehensive model, and should be used henceforth replacing the existing model.

ix. The present work proposed a modified model for common visualization of the propagation of nerve action potentials in a myelinated nerve fibre in which the zig-zag path between the axonal membrane and the extracellular fluid is shown (Figure 11). The author suggests that this model should replace the old model (Figure 1) in which the node of Ranvier is shown open to the extracellular fluid.

Thus the proposed mechanism based on the resistance offered to ion movement by the gaps of the interdigitated protrusions of the myelin sheaths coming from the two sides of a node of Ranvier could explain the experimental observations on stretching out of myelinated nerve fibres reasonably well with logical arguments, electrical models and mathematical analysis.

Most discoveries come from the difficulty in explaining some experimental observations and the present work also has the same history. Starting from a difficulty in explaining an observed change in nerve conduction due to head bending this present work has come up with the identification of a mechanism of nerve conduction in peripheral myelinated nerves that has remained obscure to the community of neuroscientists over almost a century of rigorous work in this very important and intriguing field. 
Again, the head bending experiment based on the new technique of distribution of F-Latency (DFL) was only a cue, but almost all of the evidence presented in this work has been taken from well-established and simple to interpret experimental findings by other scientists on ulnar nerve conduction. However, these latter findings gives a credibility to the DFL.

That conduction velocity in peripheral myelinated nerves increases due to stretching out and that it can occur immediately was something unknown to the neuroscientists. It is clear that the above experimental findings can only be explained on the basis of $R_{n e}$ as proposed in the present work. This explanation, however, does not contradict the age old concepts that conduction velocity depends directly on the diameter of a nerve fibre. So the present work gives a fuller explanation of neural conduction mechanisms that no other existing explanations offer.

Since the sources of delay for propagation of action potential have changed through this work, all relevant parameters may have to be evaluated afresh through a rigorous study. With the addition of the new model proposed and established through experiments in this work, the neural behaviour to various physical manouvres can now be studied with more confidence which may lead to new designs of experiments and may reveal new features and properties of the nervous system.

\section{ACKNOWLEDGEMENTS}

The author acknowledges the students who did the previous work on nerve conduction studies under his guidance over decades that has led to this new discovery in neuroscience. The author also acknowledges International Science Programme of Uppsala University, which has been supporting the nerve conduction studies programme of the author's group since 2011.

\section{REFERENCES}

Alam, MJ and Rabbani, KS 2010, Possible detection of cervical spondylotic neuropathy using Distribution of F-latency (DFL), a new neurophysiological parameter. BMC Research Notes, 3, 112, https://doi.org/10.1186/1756-0500-3-112.

Basser, PJ 2004, Scaling laws for myelinated axons derived from an electrotonic core-conductor model. Journal of Integrative Neuroscience. 3, 227-244.

Brown, AM and Hamann, M 2014, Computational modeling of the effects of auditory nerve dysmyelination, Front Neuroanat, https://doi.org/10.3389/fnana.2014.00073

Camizu, Accessed June 2017. https://www.camizu.org/cross-section-of-nerve/. 
Chowdhury, EA, Hussain, MJ, Rahman, MO and Rabbani, KS 2014, A double blind study to evaluate the efficacy of distribution of F- latency (DFL) in the detection of cervical radiculopathy and myelopathy, Bangladesh Journal of Medical Physics, 7, 46-55.

Classroom.sdmesa.edu, Accessed June, 2017.

http://classroom.sdmesa.edu/anatomy/images/Nervous_label/neuron2_axon_label.jpg ,

Gasser, HS and Grundfest, H 1939, Axon diameters in relation to the spike dimensions and the conduction velocity in mammalian A fibers, American Journal of Physiology, 127, 393-414. https://doi.org/10.1152/ajplegacy.1939.127.2.393

Harding, C and Halar, E 1983, Motor and sensory ulnar nerve conduction velocities: effect of elbow position. Arch. Phys. Med. Rehabil, 64(5), 227-232.

Hodgkin, AL and Huxley, AF 1952, A quantitative description of membrane current and its application to conduction and excitation in nerve, J. Physiol, 117, 500-544.

Hursh, JB 1939, Conduction velocity and diameter of nerve fibers. American Journal of Physiology, 127(1), 131-139. https://doi.org/10.1152/ajplegacy.1939.127.1.131

Keynes RD, Aidley DJ and Huang CLH, 2011. Nerve and Muscle, $4^{\text {th }}$ edition, Cambridge University Press ISBN: 9780521519557

Kimura J, Electrodiagnosis in diseases of nerve and muscle: Principles and practice. 2nd Ed. F.A. Davis company, Philadelphia, (1989).

Kolaric, KV, Thomson, G, Edgar, JM and Brown, AM 2013, Focal axonal swellings and associated ultrastructural changes attenuate conduction velocity in central nervous system axons: a computer modeling study, Physiological Reports, 1 no. e00059 (2013). DOI: 10.1002/phy2.59

Ladenbauer, J, Minassian, K, Hofstoetter, US and Rattay, F 2010, Stimulation of the human lumbar spinal cord with implanted and surface electrodes: a computer simulation study, IEEE Transactions on Neural Systems and Rehabilitation Engineering, 18(6), 637-645.

McIntyre, CC, Richardson, AG and Grill, WM 2002, Modeling the excitability of mammalian nerve fibers: influence of afterpotentials on the recovery cycle, Journal of Neurophysiology, 87, 9951006. doi: 10.1152/jn.00353.2001.

Porter, KR and Bonneville, MA 1973, Fine Structure of Cells and Tissues, 4th ed., Lea \& Febiger, Philadelphia, p. 184.

http://www.columbia.edu/itc/hs/medical/sbpm_histology_old/lab/lab03_micrograph.html.

Rabbani, KS 2011, Hypotheses to explain the occurrence of multiple peaks of DFL in nerve conduction measurement, Bangladesh Journal of Medical Physics 4, 27-36.

Rabbani, KS, Alam, MJ and Salam, MA 2007, Frequency Distribution of F-Latencies (DFL) has physiological significance and gives Distribution of Conduction Velocity (DCV) of motor nerve fibres with implications for diagnosis. Journal of Biological Physics (Springer), 33, 291-303.

Rabbani, KS, Yassin, N and Lo, YL 2014, Identification of cervical radiculo-myelopathy using Distribution of F-Latency (DFL), a new nerve conduction parameter, Bangladesh Journal of Medical Physics, 7, 34-45.

Rahman MM, 2008. Study of the distribution of F-latency (DFL) for different postures of our head and neck, MS Thesis, Department of Physics, University of Dhaka, Bangladesh. 
Rahman, MO, Chowdhury, EA and Rabbani, KS 2013, Improvement in the detection of Cervical Spondylotic Neuropathy through a combination of shifted frequency Distribution of F-Latency (DFL), Bangladesh Journal of Medical Physics, 6, 75-81.

Ritchie, JM, 1995, Physiology of axons in Axon, structure and function and pathophysiology, Eds: Waxman SG, Kocsis JD and Stys PK, Ch. 4, p 79.

Sanders FK, 1948. The Thickness of the Myelin Sheaths of Normal and Regenerating Peripheral Nerve Fibres, Proceedings of the Royal Society of London. Series B, Biological Sciences. 135 (880) 323-357.

Sattari, S and Emad, M R 2007, Changes in ulnar nerve conduction velocity across the elbow in different angles of elbow flexion. Electromyogr. Clin. Neurophysiol, 47(7-8), 373-376.

Sharmin, S and Rabbani, KS, 2016. Changes in the Distribution of F-latency (DFL) for different postures of head and neck. Bangladesh Journal of Medical Physics 9, 1-10.

Thoirs, K, Williams, M and Phillips, M 2008, Ultrasonographic measurements of the Ulnar Nerve at the Elbow: Role of Confounders. J. Ultrasound Med, 27, 737-743. DOI: 10.7863/jum.2008.27.5.737. 